\title{
Construção e avaliação de um jogo educacional digital de Modelagem de Dados para o ambiente universitário
}

\author{
Stela Sampaio Silva ${ }^{1}$, Angelica Toffano Seidel Calazanss, Bruno Magno da Silva ${ }^{1}$ \\ Curso de Análise e Desenvolvimento de Sistemas - Centro Universitário de Brasília \\ (UniCEUB) - Brasília ${ }^{1}$ \\ 70790-075 - Brasília- DF- Brasil \\ stelasampaiosegmail.com, \\ angelica toffanodyahoo.com.br,brunno.magnno@gmail.com
}

\begin{abstract}
The purpose of this research was to develop and evaluate an educational game for the Data Modelling discipline in System Analysis and Development at UniCEUB, to add diversity to the discipline and improve the skills and knowledgement of the students. The game was developed in 4 stages: pre-production, production, testing and post-production. Java, JavaScript with Bootstrap, C\#, PHP and MySQL database were used. The game evaluation followed the Savi et al. model, and the Goal-QuestionMetrics approach to metrics. The game was evaluated by 22 students and results show that: it increased motivation, was regarded as a positive experience and contributed to learning.
\end{abstract}

Resumo. O objetivo foi desenvolver e avaliar um jogo digital educativo de Modelagem de Dados para o curso de Análise e Desenvolvimento de Sistemas do UniCEUB, visando agregar diversidade à disciplina e aprimorar as habilidades e conhecimentos dos alunos. Para produção do jogo foram seguidas 4 fases: pré-produção, produção, testes e finalização. Foram utilizadas as ferramentas JAVA, JavaScript e Bootstrap, C\# e PHP e banco de dados MySQL. A avaliação do Jogo foi realizada com base no modelo de Savi et AL, e para construção das métricas utilizou-se o Goal-Question-Metrics. $O$ jogo foi avaliado por 22 alunos e foi identificado que: proporcionou motivação, foi uma experiência positiva e contribuiu para aprendizagem.

\section{Introdução}

Os jogos digitais tornaram-se uma forma de entretenimento muito usada tanto por crianças, quanto por jovens e adultos. Esses jogos normalmente criam desafios aos jogadores, os quais devem desenvolver uma maneira de solucioná-los, fazendo com que passem pelo ciclo de observação, questionamento, hipótese e teste (DE FREITAS, 2006). Esse ciclo os estimulam a desenvolverem habilidades como raciocínio lógico, raciocínio estratégico, planejamento, comunicação e capacidade para tomada de decisões (KIRRIEMUIR \& MCFARLANE, 2004).

Teorias modernas sobre aprendizado sugerem que o aprendizado é mais efetivo quando envolve uma atividade experimental, com resolução de problema e com um feedback imediato (BOYLE, CONNOLLY \& HAINEY, 2011). Os jogos podem proporcionar essa capacidade de aprendizado, além de serem um meio de 
entretenimento, por isso, passaram a ser usados em ambientes educacionais, como escolas, universidades e em grandes empresas (BOZSIK, 2013).

Os jogos digitais são usados no ambiente acadêmico por serem mais simples e fáceis de serem produzidos do que os jogos eletrônicos, apesar de sua jogabilidade e realismo ser semelhante (ANTONIETTI, 2002; MIGLINO, 2007). Considerando esses aspectos, alguns questionamentos foram elaborados: os jogos digitais podem facilitar o aprendizado de estudantes que cursam disciplinas muito teóricas com assuntos de alta abstração? Em cursos de graduação como Análise e Desenvolvimento de Sistemas de Informação e Ciências da Computação, um jogo digital pode melhorar o aprendizado, a habilidade dos alunos e tornar o ensino mais agradável?

Para responder a essas questões, a disciplina escolhida para o desenvolvimento do jogo foi a modelagem de dados. As pesquisas realizadas não identificaram a existência de um jogo digital para esse contexto. E foi definido o objetivo geral dessa pesquisa que é construir e avaliar um jogo digital de modelagem de dados para o ambiente universitário, visando agregar mais diversidade ao ensino da disciplina e aprimorar as habilidades e conhecimentos dos alunos.

E são os seguintes objetivos específicos:

1. Identificar e analisar as metodologias para o desenvolvimento de jogos digitais educativos;

2. Pesquisar e analisar as ferramentas para construção de um jogo digital educativo;

3. Construir um jogo digital para o ambiente universitário;

4. Avaliar os aspectos positivos e pontos a melhorar da utilização desse jogo no ambiente universitário;

Para atender a esses objetivos apresenta-se, na seção 2, uma breve descrição dos jogos educacionais focados no ensino superior. Na seção 3, apresenta-se a Metodologia da pesquisa e da produção do jogo, e a análise dos resultados obtidos são apresentados na seção 4. Finalmente, as conclusões são apresentadas na seção 5.

\section{Jogos digitais educacionais para o ensino superior}

O jogo digital educativo possui uma função lúdica e educativa, sendo que ambas devem coexistir em equilíbrio. O envolvimento ativo do estudante em um jogo educativo acontece através de ações utilizadas nos jogos que provoquem o aluno a interagir com elementos novos, possibilitando que este elabore respostas aos problemas suscitados, superando as contradições entre a sua representação e a realidade (VASCONCELLOS, 2002).

No contexto de jogos universitários focados na área de análise e desenvolvimento de sistemas e/ou Ciência da computação, identificam-se algumas propostas, tais como: Ilha de Requisitos (THIRY, ZOUCAS \& GONÇALVES, 2010), Re-O-Poly (RENEL \& GOTEL, 2008), U-Test (SILVA,2010), SPI City (SILVEIRA, THIRY \& ZOUCAS, 2013).

Não foram encontrados jogos focados na disciplina de modelagem de dados para cursos de Engenharia de software e Análise e desenvolvimento de sistemas. A disciplina 
de Modelagem de dados possui carga horária de 75 horas e tem como objetivo "Introduzir os conceitos fundamentais da natureza e do propósito da modelagem de dados na construção de sistemas de informação, dando ênfase em modelagem relacional”. A seguir apresenta-se a metodologia da pesquisa.

\section{Metodologia da pesquisa}

Essa investigação utilizou a abordagem qualitativa e quantitativa. No que tange ao alcance temporal, a pesquisa se caracterizou como uma pesquisa interseccional, uma vez que investigou as metodologias existentes para desenvolvimento de jogos digitais educativos e as ferramentas para construção de um jogo digital educativo. Além disso, foi construído um jogo e realizada sua avaliação em determinado período.

O estudo foi baseado no levantamento documental sobre jogos digitais, metodologia, ferramentas, sua aplicação e avaliação no contexto universitário. Foram seguidos os passos definidos por Chandler (2012) para construção do jogo: préprodução, produção, testes e finalização.

O método adotado para avaliação das vantagens e desvantagens da aplicação desse jogo no ambiente universitário foi o estudo de caso. Segundo Wangenheim et al. (2009), é necessário avaliar a efetividade dos jogos como ferramentas de aprendizagem. A avaliação do Jogo de Modelagem de Dados (JMD) foi realizada com base no modelo específico para jogos educacionais proposto por Savi et al. (2011). Esse modelo é baseado no modelo de avaliação de programas de treinamento de Kirkpatrick (1994) e nas estratégias motivacionais do modelo ARCS (KELLER, 1987).

Segundo Savi et al. (2011), o modelo possui o foco em jogos que possam ser utilizados como material educacional para apoiar o processo de ensino e aprendizagem da Engenharia de Software, avaliando se o jogo motiva os estudantes a utilizarem a ferramenta como material de apoio, se proporciona experiência positiva com os jogadores e principalmente se o jogo educativo proporciona o conhecimento para o conceito relacionado. Os itens da escala de avaliação foram divididos em três subescalas: Motivação, Experiência do Usuário e Aprendizagem. O modelo propõe o uso de questionário para avaliar as subescalas.

O questionário foi dividido em duas partes: A primeira parte utiliza o formato de resposta dos itens em uma escala que vai de -2 até +2 . Uma nota -2 indica forte discordância com um item e +2 forte concordância. A segunda parte avalia mais pontualmente a aprendizagem, através de itens relacionados aos objetivos de aprendizagem. Nessa avaliação considera-se uma escala de 1 até 5. E o usuário avalia a sua percepção sobre o objetivo de aprendizagem antes e após o jogo. Para essa avaliação foram considerados dois objetivos de aprendizagem: Aprendizagem com relação aos conceitos de entidades, atributos, identificadores e Aprendizagem com relação aos conceitos de relacionamentos e cardinalidades.

Para construir as métricas e indicadores para avaliação do jogo e dos objetivos de aprendizagem, utilizou-se o GQM (Goal-Question-Metrics) (BASILI et al., 1994), conforme a Tabela 1. A abordagem GQM é utilizada sobre a suposição de que para medir de forma objetiva, deve-se especificar quais os objetivos a serem alcançados com as medições estabelecidas. Tais objetivos direcionam a elaboração de questões que, 
depois de refinadas, resultam em métricas, cuja aplicação responderá as questões estabelecidas e, consequentemente, os objetivos de medição identificados (BASILI et al., 1994). A Tabela 1 apresenta o Objetivo do estudo segundo o GQM e a Tabela 2 apresenta parte das questões avaliadas com as medidas e indicadores criados.

Tabela 1: Objetivo do estudo segundo o GQM

\section{Objetivo 1: Analisar o Jogo de Modelagem de Dados}

Propósito: caracterizar

Em relação a: Efetividade de Aprendizagem, Motivação e Experiência do Usuário

Do ponto de vista: dos alunos

No contexto da: disciplina de Modelagem de dados do Curso de Análise e desenvolvimento de sistemas

Tabela 2: Questões avaliadas de efetividade de aprendizagem dos conceitos de entidade, atributos, identificadores e cardinalidades

\begin{tabular}{|c|c|}
\hline \multicolumn{2}{|c|}{$\begin{array}{l}\text { Questão 1.1: Qual a efetividade da aprendizagem dos conceitos de entidades, } \\
\text { atributos, identificadores, relacionamentos e cardinalidades? }\end{array}$} \\
\hline Métrica 1.1a & $\begin{array}{l}\text { O objetivo dessa métrica é mensurar a efetividade da } \\
\text { aprendizagem com relação aos conceitos de entidades, atributos, } \\
\text { identificadores, relacionamentos e cardinalidades }\end{array}$ \\
\hline Classificação & Objetiva e quantitativa \\
\hline $\begin{array}{l}\text { Base } \\
\text { medição }\end{array}$ & $\begin{array}{l}\text { Relação entre a nota dada para cada questão e o quantitativo de } \\
\text { alunos que avaliaram } \\
\text { - Questões do questionário } 25,26 \text { e } 27 \text { - Percepção dos alunos }\end{array}$ \\
\hline Medidas & $\begin{array}{l}\text { Para cada uma das questões } 25,26 \text { e } 27 \\
\text { M1.1a_1 }=\left(\sum \text { qtd forte discordância/ } / \text { qtd respostas }\right) * 100 \\
\text { M1.1a_2 }=\left(\sum \text { qtd discordância/ } \sum \text { qtd respostas }\right) * 100 \\
\text { M1.1a_3 }=\left(\sum \text { qtd concordância/ } / \text { qtd respostas }\right) * 100 \\
\text { M1.1a_4 }=\left(\sum \text { qtd forte concordância/ } / \text { qtd respostas }\right) * 100\end{array}$ \\
\hline Indicador & $\begin{array}{l}\text { (M1. 1a_3 + M1.1a_4) para cada questão (25,26 e } 27) \\
\text { - de } 50 \% \text { a } 70 \% \text { (o jogo contribuiu para a aprendizagem) } \\
\text { - acima de } 70 \% \text { (o jogo contribuiu muito para aprendizagem) }\end{array}$ \\
\hline
\end{tabular}

Para avaliar os objetivos de aprendizagem foi utilizado o teste t de Student para amostras pareadas. O objetivo foi comparar as MÉDIAS DE DUAS distribuições normais, supondo que se trata da MESMA população, mas em dois momentos diferentes: antes e após a execução do jogo. Segundo Barbetta (2002), "o teste t é apropriado para comparar dois conjuntos de dados quantitativos, em termos de seus valores médios". Esse teste permite identificar se a tendência identificada poderia ser explicada apenas por efeitos causais ou não.

\section{Produção do jogo e análise dos resultados}

Para produção do jogo, foi utilizado o ciclo básico proposto por Chandler composto de 4 fases: pré-produção, produção, testes e finalização. Assim, na fase de pré-produção foram definidos objetivos, mecânica do jogo, áreas, entre outros. A história do jogo se inicia com a personagem principal indo para um mundo dos sonhos quando adormece em uma aula de modelagem de dados, neste mundo ela deve enfrentar 
desafios que surgem em seu caminho e adquirir conhecimento. A arte conceitual do jogo é baseada em jogos dos anos 80, como Super Mario Bros e Alex Kidd.

A mecânica do jogo foi baseada na curva de aprendizado do jogador e no decorrer do jogo, as questões aumentam de nível assim como a dificuldade do próprio cenário. O jogador é recompensado sempre que acerta uma questão, demonstrando seu aprendizado durante o jogo, enquanto encontra itens de conhecimento e questões que deve responder sobre a disciplina de modelagem de dados. O conceito do jogo é baseado na aquisição de pontos, ou seja, a missão do jogador é adquirir o maior número possível de pontos acertando as questões relacionadas à disciplina de modelagem de dados $\mathrm{e}$ finalizar todas as fases do jogo sem ser derrotado pelos inimigos.

O jogo possui três áreas: Área do Administrador que tem o layout completo do sistema, com todas as opções de botões. Foi desenvolvido utilizando a biblioteca Bootstrap; Área do professor composta pelas opções de manutenção das questões do jogo e da geração de relatórios que não existem na área do aluno; Área do aluno que é composta apenas pelas opções de ação de jogar o jogo e alterar senha. Na manutenção das questões do jogo o professor pode inserir questões tipo exercícios (objetivas) ou questões tipo estudo de caso, onde cabe ao aluno montar o Modelo Entidade Relacionamento - MER derivado do estudo de caso proposto.

O jogo foi construído em duas interfaces: a primeira interface engloba o cadastro de questões e a administração do ambiente (área do professor e do administrador). Essa interface foi desenvolvida em JAVA, JavaScript e Bootstrap. A segunda engloba o jogo (área do aluno), onde foram implementados tanto as questões tipo exercício quanto as de estudo de caso, e foi desenvolvida em C\# e PHP. As duas interfaces utilizam o banco de dados MySQL. A Figura 1 apresenta uma das telas do jogo e a Figura 2 um exemplo do tipo de exercício estudo de caso.

Após o jogo ter sido considerado finalizado, foi implementado em duas turmas do primeiro semestre de 2015 nas disciplinas de modelagem de dados e banco de dados do curso de Análise e desenvolvimento de sistemas do UniCEUB. Apesar dos testes, o jogo apresentou algumas pequenas falhas encontradas pelos alunos enquanto jogavam durante a implantação, o que pode ter causado uma desmotivação de alguns jogadores de continuarem o jogo. No entanto, 22 alunos finalizaram o jogo. Foi possível observar os seguintes resultados de acordo com as subescalas avaliadas:

Subescala Motivação: De modo geral, observa-se que o jogo teve um efeito positivo na motivação dos alunos em grande parte dos itens, como pode ser observado no gráfico de frequências da Figura 3. O jogo foi considerado motivador por 87,02\% dos alunos, que atribuíram notas +1 ou +2 aos itens desta subescala. Sendo que $100 \%$ dos alunos consideram que foi por se esforçarem que conseguiram avançar no jogo, que atribuíram notas +1 ou +2 a este item, e $95,24 \%$ consideram que o conteúdo do jogo é relevante para os interesses deles. No entanto, o item que avalia o design do jogo não teve um nível de concordância tão alto, apenas $61,9 \%$, indicando que há espaço para melhorias nesse aspecto do jogo. 
Figura 1. Ex. da Interface do jogo JMD

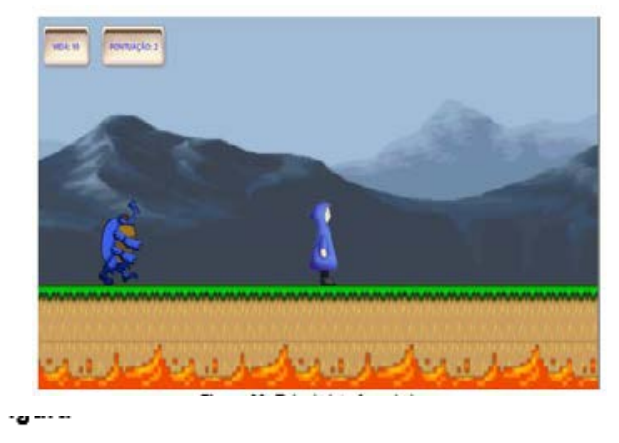

Figura 2. Ex. questão estudo de caso

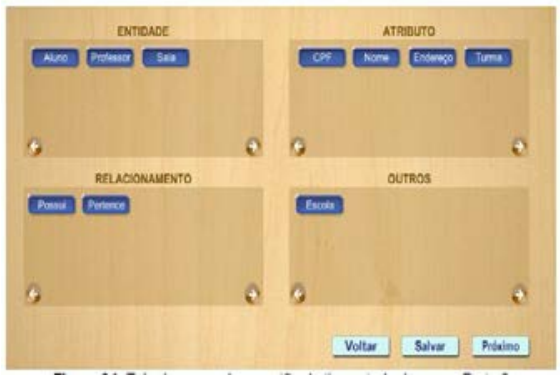

Subescala Motivação

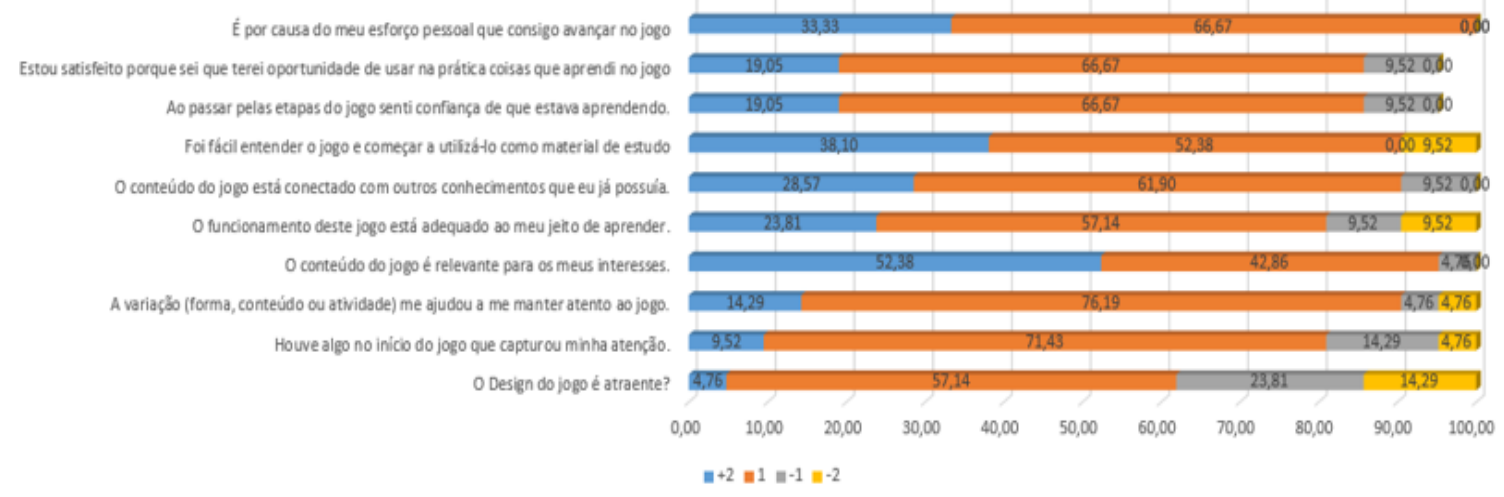

Figura 3. Gráfico de frequências da Subescala Motivação

Subescala Experiência - Observa-se que o jogo proporcionou uma experiência positiva aos alunos, conforme Figura 4. Foi considerado que o jogo proporciona uma experiência ao usuário por $54,39 \%$ dos alunos, que atribuíram notas +1 ou +2 aos itens desta subescala. $\mathrm{O}$ item melhor avaliado foi o item de dificuldade do jogo, 85,71\% dos alunos consideraram que o jogo era adequadamente desafiador sem tarefas muito fáceis ou muito difíceis, atribuindo notas +1 ou +2 a este item, e 71,43\% consideram que o jogo evolui em um ritmo adequado, não ficando monótono. Já o item que pergunta se os alunos se sentiram mais no ambiente do jogo do que no mundo real não teve o mesmo grau de concordância, $14,29 \%$ dos alunos deram notas +1 ou +2 e a faixa de notas abaixo de zero ficou em 80,96\%. Assim como este item, os outros itens sobre imersão no jogo tiveram notas mais baixas, sendo que apenas $41,85 \%$ não perceberam o tempo passar enquanto jogavam e $42,86 \%$ não conseguiram esquecer suas preocupações do dia-a-dia durante o jogo.

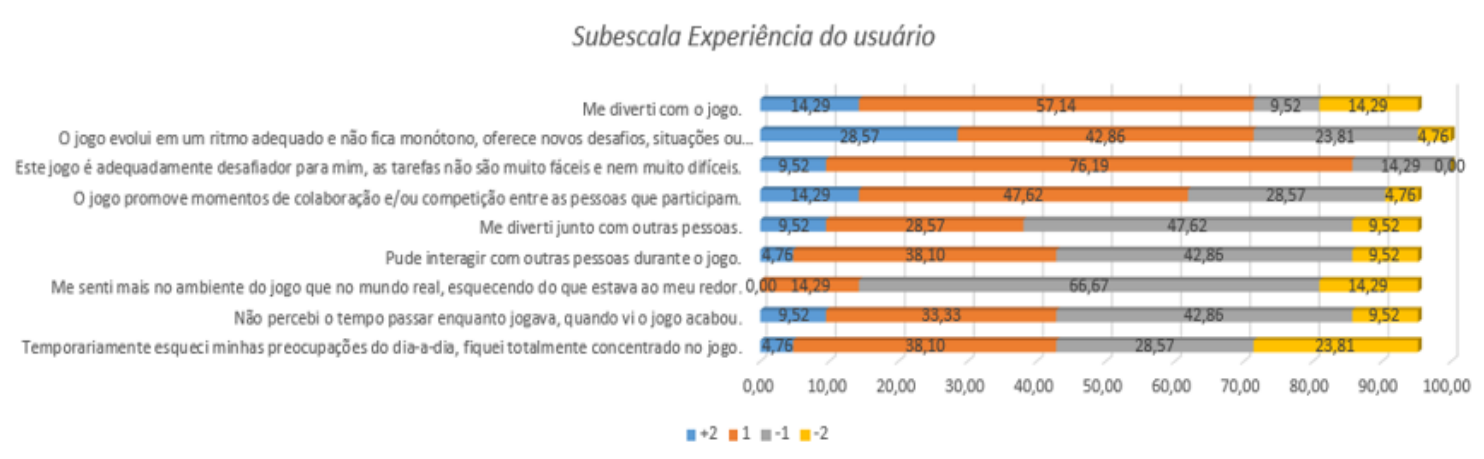

Figura 4. Gráfico de frequências da Subescala Experiência 
Subescala Aprendizagem - Ao observar as respostas dos itens indicadores de efetividade da aprendizagem, o jogo contribuiu para a aprendizagem dos alunos, como pode ser observado no gráfico da Figura 5 . Sendo que $85,24 \%$ dos alunos atribuíram notas +1 ou +2 aos itens desta subescala, consideraram que o jogo contribui para sua aprendizagem em relação aos conceitos de entidade, atributo, relacionamento, cardinalidade e identificadores da disciplina de modelagem de dados. Houve um nível de $85,72 \%$ de forte concordância de que o jogo foi eficiente para a aprendizagem, quando comparado com outras atividades da disciplina, com $71,43 \%$ de notas +1 e $14,29 \%$ de notas +2 , e $85,71 \%$ dos alunos consideram que o jogo contribuiu para a aprendizagem da disciplina. $\mathrm{O}$ item com a menor nota teve uma concordância de $76,19 \%$ dos alunos que consideram que a experiência com o jogo vai contribuir com seu desempenho na vida profissional.

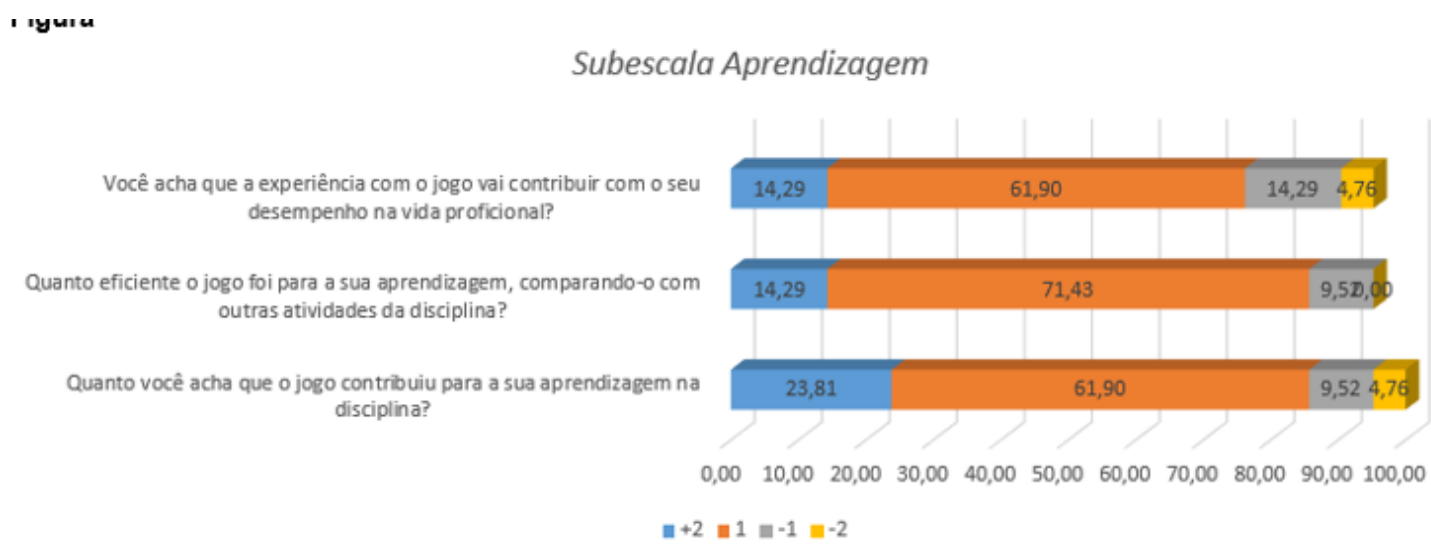

Figura 5. Gráfico de frequências da Subescala Aprendizagem

A Tabela 3 e a Figura 6 apresentam as médias da auto-avaliação dos alunos em relação aos objetivos de aprendizagem nos momentos antes e depois do jogo com base no modelo de Savi et al. (2011). Os resultados foram obtidos por teste t de Student para amostras pareadas. O objetivo foi comparar as MÉDIAS DE DUAS distribuições normais, supondo que se trata da MESMA população, mas em dois momentos diferentes: antes e após a execução do jogo. É possível constatar que, os alunos perceberam um aumento de nível de conhecimento em todos os objetivos de aprendizagem do jogo. E os maiores saltos ocorreram nos itens:

- Lembrar Conceitos modelo conceitual (entidade, atributos, identificadores)

- Compreender Conceitos modelo conceitual (entidade, atributos, identificadores)

- Aplicar Conceitos modelo conceitual (entidade, atributos, identificadores)

- Compreender Conceitos modelo conceitual (relacionamento e cardinalidades)

Em todos os tipos de testes t é necessário relatar o valor de " $\mathrm{t}$ " chamado de razão crítica assim como do p-valor, pois assim pode-se identificar a veracidade da sua probabilidade $(\mathrm{p})$. Com essas informações pode-se afirmar se a diferença ocorre na realidade $(p<0,05)$ ou se existe apenas ao acaso $(p>0,05)$. Na população examinada foi identificado $\mathrm{p}<0,05$ nos itens listados a seguir, demonstrando dessa forma que, para esses itens, a diferença entre a aprendizagem nos momentos antes e depois do jogo ocorreu na realidade, considerando a população estudada: 
2. Compreender Conceitos modelo conceitual (entidade, atributos, identificadores) 0,0373925000135277

3. Aplicar Conceitos modelo conceitual (entidade, atributos, identificadores) 0,0261449195292569

Tabela 3. Médias da auto-avaliação dos alunos em relação aos objetivos de aprendizagem nos momentos antes e depois

\begin{tabular}{|c|c|c|c|c|}
\hline Perguntas & Momento & Média & Varianoia & $\begin{array}{l}\text { Diferenç } \\
\text { a antes- } \\
\text { depois }\end{array}$ \\
\hline $\begin{array}{c}\text { Lembrar Conceitos } \\
\text { modelo conceitual } \\
\text { [entidade, atributos, } \\
\text { identificadores] }\end{array}$ & \begin{tabular}{|l} 
Antes \\
Depois \\
\end{tabular} & $\begin{array}{r}3,4 \\
3,85 \\
\end{array}$ & $\begin{array}{r}0.98947 \\
0,87105\end{array}$ & 0,45 \\
\hline $\begin{array}{c}\text { Comprasnotor } \\
\text { Conceitos modelo } \\
\text { oonceitual (entidade, } \\
\text { atributos. } \\
\text { identificadores) }\end{array}$ & \begin{tabular}{|l} 
Antes \\
Depois \\
\end{tabular} & $\begin{array}{r}3,3 \\
3,85 \\
\end{array}$ & $\begin{array}{r}0,85263 \\
0,87105 \\
\end{array}$ & 0,55 \\
\hline $\begin{array}{c}\text { Aolioar Conceitos } \\
\text { modelo oonoeitual } \\
\text { [entidade, atributos, } \\
\text { identificadores] }\end{array}$ & \begin{tabular}{|l} 
Antes \\
Depois
\end{tabular} & $\begin{array}{r}3,47 \\
3,945\end{array}$ & $\begin{array}{l}0,98642 \\
0,99734\end{array}$ & 0,475 \\
\hline $\begin{array}{c}\text { Lembrar Conceitos } \\
\text { modelo oonceitual } \\
\text { (relacionamento } \\
\text { cardinalidades] }\end{array}$ & \begin{tabular}{|l} 
Antes \\
Depois \\
\end{tabular} & $\begin{array}{r}3,7325 \\
3,895 \\
\end{array}$ & $\begin{array}{r}0,7206 \\
0,93629 \\
\end{array}$ & 0,1625 \\
\hline $\begin{array}{c}\text { Compnasneter } \\
\text { Conceitos modelo } \\
\text { conoeitual } \\
\text { (relacionamento e } \\
\text { cardinalidades]. }\end{array}$ & \begin{tabular}{|l} 
Antes \\
Depois
\end{tabular} & $\begin{array}{r}3,55 \\
3,9\end{array}$ & $\begin{array}{l}0,68158 \\
0,83158\end{array}$ & 0,35 \\
\hline $\begin{array}{l}\text { Afolivar Conceitos } \\
\text { modelo oonoeitual } \\
\text { [relacionamento e } \\
\text { cardinalidades] }\end{array}$ & \begin{tabular}{|l} 
Antes \\
Depois \\
\end{tabular} & $\frac{3,3}{3,6}$ & $\begin{array}{r}0.95789 \\
1,2\end{array}$ & 0,3 \\
\hline
\end{tabular}

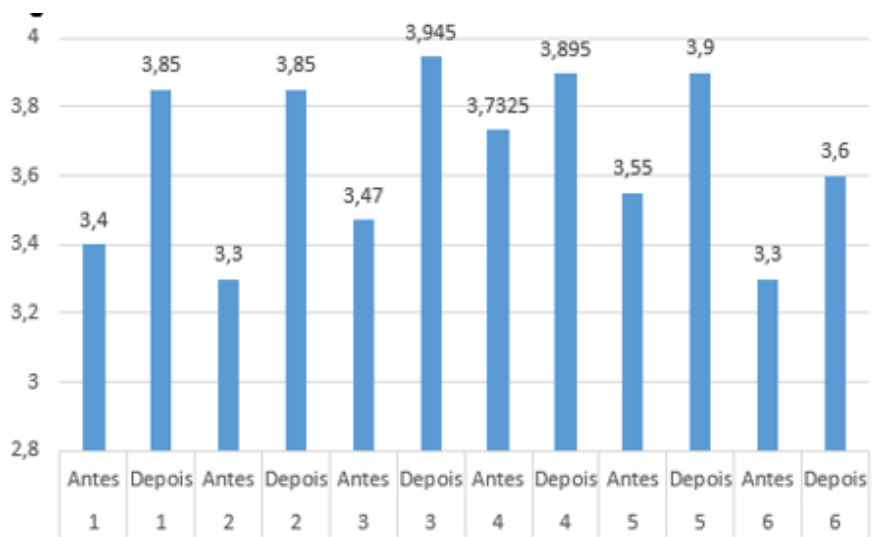

Figura 6. Médias da auto-avaliação dos alunos em relação aos objetivos de aprendizagem nos momentos antes e depois do jogo

Legenda:

1. Lembrar Conceitos modelo conceitual (entidade, atributos, identificadores)

2. Compreender Conceitos modelo conceitual (entidade, atributos, identificadores)

3. Aplicar Conceitos modelo conceitual (entidade, atributos, identificadores) 
4. Lembrar Conceitos modelo conceitual (relacionamento e cardinalidades)

5. Compreender Conceitos modelo conceitual (relacionamento e cardinalidades)

\section{Conclusões e estudos futuros}

$\mathrm{Na}$ educação, os docentes encontram cada vez mais alternativas na tecnologia para melhorar a forma como repassar conhecimentos e desenvolver as habilidades de seus alunos. Os jogos digitais surgiram como uma forma de entretenimento entre jovens no início da popularização dos computadores e videogames, mas esta realidade tem mudado nos últimos anos e os jogos passaram a serem vistos como uma forma de auxiliar professores em sala de aula.

Este trabalho teve como objetivo desenvolver e avaliar um jogo digital educativo de Modelagem de Dados para o curso de graduação em Tecnologia de Análise e Desenvolvimento de Sistemas visando agregar mais diversidade ao ensino da disciplina e aprimorar as habilidades e conhecimentos dos alunos.

Para atender aos objetivos geral e específicos foram pesquisados os jogos educacionais para ambiente universitário e a aplicabilidade do jogo em disciplinas do Curso de Análise e Desenvolvimento de Sistemas. Foram pesquisadas as metodologias e ferramentas existentes para a criação de um jogo. Após essas pesquisas, foi definido o foco do jogo como sendo a disciplina de Modelagem de Dados e a utilização do ciclo básico proposto por Chandler para produção do jogo, composto de 4 fases: préprodução, produção, testes e finalização. Foram utilizadas as ferramentas já citadas e o jogo foi avaliado na percepção de 22 alunos da disciplina considerando as dimensões de Experiência, Motivação e Aprendizagem. Os resultados foram promissores e foram identificados alguns aspectos de melhorias.

Como trabalhos futuros, é interessante que novas turmas de modelagem de dados utilizem o jogo e o avaliem novamente, uma vez que o jogo, foi evoluído no 2o. semestre de 2015 para uma nova release com implementações de melhorias em suas funcionalidades e na interface. Além disso, será interessante também realizar avaliações de desempenho (com a ferramenta e sem a ferramenta) para tentar mensurar os ganhos com a mesma.

\section{Referências}

Antonietti, A., Rasi, C. and Underwood, J.. I videogiochi: una palestra per il pensiero strategico? "Rivista: Ricerche di Psicologia". Fascicolo: 1, p.125-144, 2002.

Barbetta, P. A. "Estatistica aplicada as ciências sociais". 5a. Ed. -Florianopolis: Ed. Da UFSC, 2002.

Basili. V., Caldiera, G.and Rombach, H. Goal Question Metric paradigm. In: "Encyclopédia of Software Engineering". V. 2, 1994. p. 527 -532.

Boyle, E. A., Connolly T. M. and Hainey, T. The role of psychology in understanding the impact of computer games. "Entertainment Computing", 2, 69-74, 2011.

Bozsik S. Business simulation game as a tool of practice-oriented educatin. "Practice and Theory in Systems of Education", Volume 8, Number 2, p. 126-134, 2013. 
Chandler, H. M. "Manual de Produção de Jogos Digitais", 2 a edição. Bookman, VitalBook file, 2012.

De Freitas, S. Learning in immersive worlds. "Joint Information Systems Committee", 2006.

Kirriemuir, J.and Mcfarlane, A. Literature Review in Games and Learning. "A NESTA Futurelab Research report” - report 8,. 2004.

Keller, J.M. Development and use of the ARCS model of instructional design. "Journal of instructional development". Volume 10, Issue 3,pp 2-10, September, 1987

Kirkpatrick, D. L.,. "Evaluating Training Programs - The Four Levels". Berrett-Koehler Publishers, 1994.

Miglino, O.; Ferdinando A. D. and Rega, A. Le nuove macchine per apprendere: simulazioni al computer, robot e videogiochi multi-utente. "Rivista Sistemi intelligenti". Fasciculo 1, aprile p. 113-135, 2007.

Renel, S. and Gotel, O. Gameplay to Introduce and Reinforce RequirementsEngineering Practices, 16th IEEE "International Requirements Engineering Conference", Barcelona, Spain, IEEE Computer Society Press. 2008.

Savi, R.; Wangenheim, C. and Borgatto, A. Análise de um modelo de avaliação de jogos educacionais. Disponível em: https://sites.google.com/site/savisites/avaliacao-de-jogoseducacionais, 2011.

Silva, A. C. "Jogo educacional para apoiar o ensino de técnicas para elaboração de testes de unidade". Dissertação (Mestrado) - Curso de Computação Aplicada, Universidade do Vale do Itajaí, São José, 195 f. , 2010.

Silveira, J. L, Thiry, M. and Zoucas, A. SPI City: Jogo Educacional para Apoiar o Ensino de Melhoria de Processo de Software.In:"XII Simpósio brasileiro de qualidade de software". Salvador, Bahia - Brasil. 01 a 05 de Julho, 2013.

Thiry, M., Zoucas, A and Gonçalves, R. Q. Promovendo a Aprendizagem de Engenharia de Requisitos de Software Através de um Jogo Educativo. In: "XXI SBIE - Simpósio Brasileiro de Informática na Educação”, 2010, João Pessoa. 2010.

Vasconcellos, C.S. "Construção do conhecimento em sala de aula". São Paulo: Libertad, 2004.

Wangenheim, C., Kochanski, D. and Savi, R. Revisão Sistemática sobre Avaliação de Jogos Voltados para Aprendizagem de Engenharia de Software no Brasil. In: "Anais do FEES 2009/ XXIII SBES”, pp. 41-48, Fortaleza, Brasil. 2009. 